\title{
A multi-omics approach to understanding the field effect in bladder cancer
}

\author{
Xue Li ${ }^{1,2}$, Ali Hashemi Gheinani ${ }^{1,2}$, Rosalyn M. Adam ${ }^{1,2}$ \\ ${ }^{1}$ Urological Diseases Research Center, Boston Children's Hospital, Boston, MA, USA; ${ }^{2}$ Department of Surgery, Harvard Medical School, Boston, \\ MA, USA \\ Correspondence to: Rosalyn M. Adam, PhD. Urological Diseases Research Center, Enders Research Building, Room 1061.3, Boston Children's \\ Hospital \& Harvard Medical School, Boston, MA 02115, USA. Email: rosalyn.adam@childrens.harvard.edu. \\ Provenance: This is an invited article commissioned by Section Editor Xiao Li (Department of Urology, Jiangsu Cancer Hospital \& Jiangsu Institute \\ of Cancer Research \& Nanjing Medical University Affiliated Cancer Hospital, Nanjing, China) \\ Comment on: Majewski T, Yao H, Bondaruk J, et al. Whole-Organ genomic characterization of mucosal field effects initiating bladder \\ carcinogenesis. Cell Rep 2019;26:2241-56.e4.
}

Submitted Jul 06, 2019. Accepted for publication Jul 12, 2019.

doi: 10.21037/tau.2019.07.11

View this article at: http://dx.doi.org/10.21037/tau.2019.07.11

The field effect in cancer is a phenomenon that describes histologically normal-appearing tissue surrounding or adjacent to a neoplasm that can give rise to additional neoplastic lesions. Bladder cancer has a high rate of recurrence and the development of multifocal lesions is common. Molecular analyses in a variety of tumor types, including bladder cancer, have implicated genetic and epigenetic alterations in so-called field cancerization $(1,2)$. Majewski and colleagues recently combined state-of-theart genomics analyses with a whole-organ histologic map to interrogate molecular changes within the urothelium of a whole bladder harboring multifocal papillary cancer (3). From a single surgical specimen, 41 mucosal samples were collected for histopathological evaluation and multi-platform genomics analyses including whole-exome sequencing (WES, 37 samples), copy number variation (CNV, 33 samples) and whole-genome DNA methylation (27 samples). The rich resource generated from the study provides novel insights into the molecular changes that drive the field effect in bladder carcinogenesis.

In this study, the authors identified a total of 386 genomic loci with non-silent mutations, which comprised 312 single-nucleotide variants ( $\mathrm{SNVs}, \mathrm{n}=312)$ and indels $(\mathrm{n}=74)$. Mutations fell into two clusters based on frequency across individual mucosal regions, with cluster ' $a$ ' mutations shared by multiple samples and showing the increased frequency with progression, and cluster ' $b$ ' mutations showing low frequency and restriction to individual samples. Hierarchical clustering of a subset of genes with non-silent SNVs or indels revealed two clusters that behaved differently with neoplastic progression. Genes in the first cluster showed increased frequency of variant alleles with evolution to high-grade intraurothelial neoplasia (HGIN) and urothelial carcinoma (UC), and included those encoding transcription factors and cell cycle regulators, cell adhesion proteins, small GTPases, and chromatin remodeling factors. In contrast, genes in the second cluster did not show altered frequency of variant alleles with progression and were enriched for those regulating protein ubiquitination, epithelial differentiation, cell proliferation and innate immunity.

To assess the clonal landscape of the field effect, the mutation pattern was assessed across the mucosa. Between 1 and 33 mutations (10, on average) were shared by all samples, but many regions displayed only unique alterations. However, there was a significant increase in private mutations shared by samples corresponding to HGIN and UCs indicating the clonality of HGIN and UC. Thus, there is significant mutational heterogeneity in the field effect together with significant alteration in the mutational landscape with disease progression. To address the evolution of the mutational signatures as the field effect progressed to $\mathrm{UC}$, the authors analyzed single base substitutions $(\mathrm{C}>\mathrm{A}, \mathrm{C}>\mathrm{G}, \mathrm{C}>\mathrm{T}, \mathrm{T}>\mathrm{A}, \mathrm{T}>\mathrm{C}$, and $\mathrm{T}>\mathrm{G})$ in all mucosal samples and identified a significant increase in the pattern for $\mathrm{C}>\mathrm{T}$ mutations that increased from 
evolution of normal urothelium/low-grade intraurothelial neoplasia (NU/LGIN) to HGIN and UC. By comparison with the Sanger signatures reported previously $(4,5)$, more than 10 distinct signatures were observed in individual samples, and were considerably more heterogeneous in the mucosal field effect corresponding to NU/LGIN than in the samples of HGIN or UCs. Among the signatures detected were those attributed to tobacco use, BRCA1/2 mutations, DNA mismatch repair, and APOBEC, as well as a number associated with unknown biological processes. Interestingly, the APOBEC signature, which has been implicated in progression of both non muscle-invasive and muscle-invasive bladder cancer (6-9), was not increased significantly with progression to HGIN and UCs, and was not prominent in the overall mutational landscape in this patient. A recent multi-region study of human bladder cancer using whole cystectomy specimens from 10 patients (10) found that APOBEC mutational signatures were present very early in the disease course and persisted as a major source of mutations throughout tumor evolution.

APOBEC activation is a component of innate immunity against viruses and retrotransposons (11). In the current study, analysis of copy number changes in mucosal samples using genome-wide $S N P$ arrays identified significant copy number gains on chromosome $7 \mathrm{q}$ and losses on chromosome 8p. Among the losses on $8 \mathrm{p}$ were loci encoding anti-microbial proteins called defensins that act in innate immunity. One locus harboring 11 genes encoding defensins was deleted early in disease and associated with clonal expansion of the field effect, whereas a separate locus encoding defensins was deleted later during the evolution of HGIN to UC. As noted above, the frequency of mutations in several genes that regulate innate immunity including FLG2, HRNR, and PIK3CB was noted to be unchanged with the evolution from NU/LGIN to HGIN to UC, and so these mutations are present at the earliest stages of neoplasia and throughout the disease course. Notably, a recent report demonstrated that exposure to uropathogenic E. coli elicited alterations in the mucosa similar to those observed upon exposure to cancer-causing nitrosamines (12). Together, these discrete findings provide evidence for a role for immune mechanisms in cancer initiation.

In addition to CNVs and mutational analysis, the authors also explored whole-genome DNA methylation changes across all mucosal samples using hybridization-based microarrays corresponding to $\sim 10,000$ gene-associated $\mathrm{CpG}$ islands. 610 genes were found to be either hyper- or hypomethylated in at least one mucosal sample by comparison with normal urothelium from the ureters of patients without urothelial neoplasia. Hierarchical clustering of genes based on methylation level yielded two clusters comprising a majority of HGIN and UC samples in one, and the majority of NU/LGIN, plus one HGIN and one UC samples in the other. By assessing methylation in relation to progression of neoplasia, from the mucosal field effect to HGIN and UC, differentially methylated genes could be divided into 3 clusters. Group 1 comprised genes that were abnormally methylated in NU/LGIN that persisted with development of HGIN and UC. Among the genes that were hypermethylated and predicted to be repressed were the defensin DEF6, NALP1, a component of the inflammasome, and SIGLEC8, which has been implicated in inflammatory processes. These observations are consistent with the relationship between immune processes and cancer initiation noted above. Group 2 consisted of genes with abnormal methylation that paralleled HGIN development and was retained in $\mathrm{UC}$, and contained transcription factors implicated in regulation of pluripotent stem cells as well as regulation of p53 activity. Genes in Group 3 were abnormally methylated only in UC samples and of these 12 out of 85 were linked to regulation of migration and invasion, consistent with acquisition of more aggressive oncogenic behaviors with disease progression.

To understand the functional significance of abnormal methylation and the consequences for target genes, 189 genes showing monotonic alteration associated with disease progression were subjected to Ingenuity Pathway Analysis. Forty-two canonical pathways were identified that could be divided into 3 groups based on enrichment with progression from NU to UC. Among the 18 pathways altered in the mucosal field effect associated with NU/ LGIN and progression to HGIN/UCs were those involving small GTPases, cell proliferation and invasion. Analysis of TCGA data validated the involvement of RboGDI, Rac and $C d c 42$ signaling in early field effects, and showed these pathways to be activated in UCs of both luminal and basal subtypes. Furthermore, pathways linked to activation of innate immunity were suppressed in a majority of luminal subtype tumors. Conversely gene set enrichment analysis showed the luminal subtype was enriched for immune suppression. Thus, the authors strengthen their conclusion that suppression of innate immunity-associated pathways plays a significant role in development of UC, and may be an early event.

As a final analysis, the authors performed integrative molecular subtyping with iCluster, a methodology that 
integrates multiple genomic data types (13) to assess the contributions of different genomic alterations such as CNVs, mutations and DNA methylation to disease progression. Three clusters were identified by iCluster. Among the genomic features evaluated, methylation was the dominant contributor to clustering, whereas CNVs and mutations contributed equally. Cluster 1 comprised all NU/ LGIN samples plus one each of HGIN and UC; Cluster 2 comprised both HGIN and UC samples; and Cluster 3 consisted of only UC samples. Thus, the enrichment for both genomic and epigenomic alterations showed a clear association with evolution from mucosal field effect to HGIN and progression to UCs.

Several aspects of this study stand out as highlights, including the whole organ multi-omics analysis and the identification of methylation as a dominant and early contributor to the mucosal field effect and to tumor initiation. This group has applied the whole-organ mapping approach in a number of prior studies to address genetic and molecular correlates of early events in bladder cancer initiation and multi-focality (14-17). This approach enables evaluation of the entire genetic and genomic landscape, particularly in the context of clonal expansion and multifocal tumors. From a bioinformatics perspective, the analysis was robust although some limitations are noted. WES was performed initially to identify variant alleles, however mutations in introns or other non-coding sequences such as microRNAs may be missed with this approach. Also, CNV analysis relied on an array-based approach, which may be limited by the resolution and coverage of the SNPS and probes on the array. Lastly, the methylation analysis relied on the use of urothelial cell suspensions from donor ureters as a non-neoplastic control, which may have confounded interpretation, given the differing embryologic origins of bladder and ureteral urothelium. However, given the scale and multi-faceted nature of the analyses performed in the study, these are minor limitations.

In the current report, the analysis was restricted to a single patient, and this was considered to be a limitation of the study. Interestingly, in an analysis by Heide and colleagues who applied multi-omics strategies to whole cystectomy specimens from 10 patients (10), APOBEC mutations were identified very early in tumor development and were found to persist in CIS lesions and throughout tumor. Similarly, at least four other studies that applied genomic analyses to isolated tumor specimens identified an APOBEC mutational signature in early stage, nonmuscle-invasive bladder cancers $(6,7,9,18)$ consolidating the association between APOBEC mutations and cancer initiation.

As noted by the authors, one limitation of the current study was the lack of RNA expression data from mucosal samples, with expression data from the Cancer Genome Atlas used to infer gene expression changes resulting from genomic and epigenomic alterations. Since TCGA data represent muscle-invasive bladder cancers, events relevant to the earliest stages of cancer initiation, and particularly those relevant to the mucosal field effect may have been missed. RNA sequencing in several prior studies has shed light on differential gene expression associated with earlystage tumors $(7,9,18,19)$. Consistently, early-stage tumors from multiple different cohorts show enrichment for the Urobasal A subtype, which is characterized by expression of urothelial differentiation markers such as uroplakins, and the transcriptional regulators GATA3, FOXA1 and PPAR . A second subtype was also evident and defined as Genomically Unstable characterized by expression of cancer stem cell markers and epithelial to mesenchymal transition-associated (EMT-associated) transcription factors. Such changes were not noted explicitly in the current study.

The heterogeneity noted by Majewski and colleagues among mucosal specimens from a single bladder provides a strong rationale to define genomic, epigenomic and transcriptomic profiles at single-cell resolution. With the ever-increasing interest and use of single cell genomics approaches in a wide variety of contexts, it is only a matter of time before these data can be integrated into current analytical pipelines.

\section{Acknowledgments}

None.

\section{Footnote}

Conflicts of Interest: The authors have no conflicts of interest to declare.

Ethical Statement: The authors are accountable for all aspects of the work in ensuring that questions related to the accuracy or integrity of any part of the work are appropriately investigated and resolved.

\section{References}

1. Curtius K, Wright NA, Graham TA. Evolution of 
premalignant disease. Cold Spring Harb Perspect Med 2017;7.

2. Czerniak B, Dinney C, McConkey D. Origins of bladder cancer. Annu Rev Pathol 2016;11:149-74.

3. Majewski T, Yao H, Bondaruk J, et al. Whole-organ genomic characterization of mucosal field effects initiating bladder carcinogenesis. Cell Rep 2019;26:2241-56.e4.

4. Alexandrov LB, Nik-Zainal S, Wedge DC, et al. Signatures of mutational processes in human cancer. Nature 2013;500:415-21.

5. Nik-Zainal S, Alexandrov LB, Wedge DC, et al. Mutational processes molding the genomes of 21 breast cancers. Cell 2012;149:979-93.

6. Nordentoft I, Lamy P, Birkenkamp-Demtröder K, et al. Mutational context and diverse clonal development in early and late bladder cancer. Cell Rep 2014;7:1649-63.

7. Hedegaard J, Lamy P, Nordentoft I, et al. Comprehensive transcriptional analysis of early-stage urothelial carcinoma. Cancer Cell 2016;30:27-42.

8. Robertson AG, Kim J, Al-Ahmadie H, et al. Comprehensive molecular characterization of muscleinvasive bladder cancer. Cell 2018;174:1033.

9. Hurst CD, Alder O, Platt FM, et al. Genomic subtypes of non-invasive bladder cancer with distinct metabolic profile and female gender bias in KDM6A mutation frequency. Cancer Cell 2017;32:701-15.e7.

10. Heide T, Maurer A, Eipel M, et al. Multiregion human bladder cancer sequencing reveals tumour evolution, bladder cancer phenotypes and implications for targeted therapy. J Pathol 2019;248:230-42.

11. Henderson S, Fenton T. APOBEC3 genes: retroviral restriction factors to cancer drivers. Trends Mol Med

Cite this article as: $\mathrm{Li} \mathrm{X}$, Gheinani $\mathrm{AH}$, Adam RM. A multiomics approach to understanding the field effect in bladder cancer. Transl Androl Urol 2019;8(6):775-778. doi:10.21037/ tau.2019.07.11
2015;21:274-84.

12. O'Brien VP, Hannan TJ, Yu L, et al. A mucosal imprint left by prior Escherichia coli bladder infection sensitizes to recurrent disease. Nat Microbiol 2016;2:16196.

13. Shen $\mathrm{R}$, Olshen $\mathrm{AB}$, Ladanyi M. Integrative clustering of multiple genomic data types using a joint latent variable model with application to breast and lung cancer subtype analysis. Bioinformatics 2009;25:2906-12.

14. Czerniak B, Chaturvedi V, Li L, et al. Superimposed histologic and genetic mapping of chromosome 9 in progression of human urinary bladder neoplasia: implications for a genetic model of multistep urothelial carcinogenesis and early detection of urinary bladder cancer. Oncogene 1999;18:1185-96.

15. Kram A, Li L, Zhang RD, et al. Mapping and genome sequence analysis of chromosome 5 regions involved in bladder cancer progression. Lab Invest 2001;81:1039-48.

16. Tuziak T, Jeong J, Majewski T, et al. High-resolution whole-organ mapping with SNPs and its significance to early events of carcinogenesis. Lab Invest 2005;85:689-701.

17. Majewski T, Lee S, Jeong J, et al. Understanding the development of human bladder cancer by using a whole-organ genomic mapping strategy. Lab Invest 2008;88:694-721.

18. Lamy P, Nordentoft I, Birkenkamp-Demtröder K, et al. Paired exome analysis reveals clonal evolution and potential therapeutic targets in urothelial carcinoma. Cancer Res 2016;76:5894-906.

19. Thomsen MBH, Nordentoft I, Lamy P, et al. Comprehensive multiregional analysis of molecular heterogeneity in bladder cancer. Sci Rep 2017;7:11702. 